\title{
Nonisothermal Device Simulation Using the 2-D Numerical Process/Device Simulator TRENDY and Application to SOI-Devices
}

Philip B. M. Wolbert, Member, IEEE, Gerhard K. M. Wachutka, Member, IEEE, Benno H. Krabbenborg, and Ton J. Mouthaan, Member, IEEE

\begin{abstract}
The electrical characteristics of modern VLSI and ULSI device structures may be significantly altered by self-heating effects. The device modeling of such structures demands the simultaneous simulation of both the electrical and the thermal device behavior and their mutual interaction. Although, at present, a large number of multi-dimensional device simulators are available, most of them are based on physical models which do not properly allow for heat transport and other nonisothermal effects.

In this paper, we demonstrate that the numerical process/device simulator TRENDY provides a solid base for nonisothermal device simulation, as a physically rigorous device model of carrier and heat transport has been incorporated in the TRENDY program. With respect to the boundary conditions, it is shown that inclusion of an artificial boundary material relaxes some fundamental physical inconsistencies resulting from the assumption of ideal ohmic contact boundaries.

The program TRENDY has been used for studying several nonisothermal problems in microelectronics. As an example, we consider an ultra-thin SOI MOSFET showing that the negative slopes in the $V_{d s}-I_{d s}$ characteristics are caused by the temperature-dependence of the electron saturation velocity.
\end{abstract}

\section{INTRODUCTION}

I $\mathrm{N}$ THE PAST, nonisothermal device simulation was particularly important for power device development. One of the first serious efforts to model and simulate nonisothermal phenomena was taken by Gaur and Navon [1]. They predicted the steady-state behavior of self-heated power transistors by solving the full system of continuity equations on a discretized 2-D domain, however, without including the temperature gradient in the constitutive current relations. Another more recent approach in simulating both electrical and thermal behavior of power devices was made by Walker et al. [2], [3], using physically sound and state-of-the-art device models. These attempts are among the few [4]-[7] that solve the full system

Manuscript received June 15, 1992. This work was supported in part by the Dutch Innovative Research Program (IOP-IC Technology). This paper was recommended by Associate Editor D. Scharfetter.

P. B. M. Wolbert was with the IC-Technology and Electronics Department, University of Twente, Enschede, The Netherlands. He is now with Philips Semiconductors B.V., MOS3-Reliability Engineering, 6534 AE Njmegen, The Netherlands.

G. K. M. Wachutka is with the Institute of Quantum Electronics, ETHHönggerberg, HPT, CH-8093 Zürich, Switzerland

B. H. Krabbenborg and T. J. Mouthaan are with the IC-Technology and Electronics Department, MESA Research Institute, University of Twente, 7500 AE Enschede, The Netherlands.

IEEE Log Number 9213667. of coupled continuity equations on a discretized general multidimensional domain. In most cases dealing with nonisothermal device modeling however, analytical methods [8], [9] or special programs [10]-[18] were used to tackle a selected problem.

Until recently, there was little interest in electro-thermal behavior from those involved in the development of VLSI devices, simply because electro-thermal effects seemed to have negligible influence on the device characteristics. However, with the continuous down-scaling of the minimum device dimensions and the emergence of more "exotic" device structures such as Silicon on Insulator (SOI), as well as with the increased sensitivity of small silicon MOSFET's to damage from Electro Static Discharge Events (ESD's), nonisothermal device simulation has become an important issue. The device characteristics may be significantly altered by electro-thermal effects. This has been reported for "normal" submicron MOSFET structures [19], [20] in the case of strong biasing, but the effect is even more pronounced in the case of ultra-thin SOI MOSFET's [21], due to the high thermal resistance of the buried oxide layer.

Local self-heating has also turned out to be a crucial effect in the operation of certain optoelectronic devices. For instance, the maximum optical output power of laser diodes, is limited by the thermal damage along the mirror facets caused by a large temperature peak having an increase of more than 100 $\mathrm{K}$ within the distance of only a few micrometers [22], [23]. It is obvious that an electro-thermal analysis of these devices will provide valuable help in finding an optimum design.

In addition to the above-mentioned problems in the field of "classical" microelectronics, we expect growing interest in electrothermal phenomena from the research and development activities in the rather novel branch of " $I C$ compatible microtransducers" [24]. Examples include thermal sensors [25], integrated cooling (Peltier) elements [26], and generally any sensor type where the measurand is transformed into an electronic signal via an intermediate thermal signal [27]. A particularly interesting subject for simulation arises from the problem of how the sensor response is affected by thermal perturbations (see, for instance, [28]), since "any sensor is a temperature sensor."

We recognize that the continuous trend towards smaller feature sizes leads to an increasing need for 3-D process and device simulation. TRENDY has been developed as a 2-D 


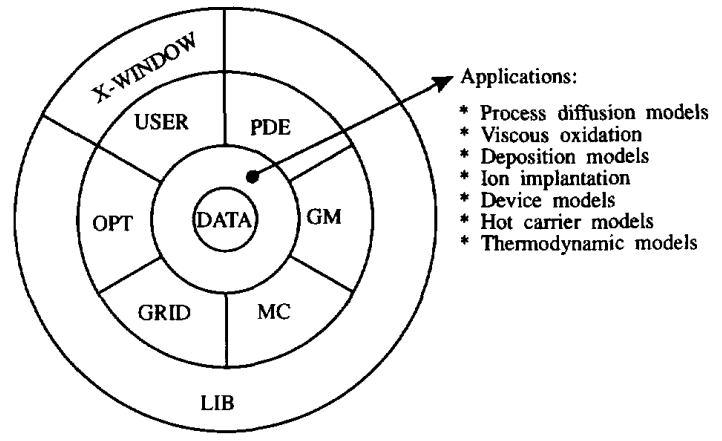

Fig. 1. Basic program organization of TRENDY.

simulator, but a future extension to 3-D has always been kept in mind. There are several reasons for the present 2D approach. First, most of today's simulation problems can still be treated by a 2-D approach without significant loss of accuracy. Second, one still struggles worldwide with the problem of accurate and reliable 2-D process simulations, which imposes a serious restriction on the practicality of 3-D device simulation programs. Third, 3-D device simulations imply large memory requirements and still take an excessive amount of CPU time, even on modern computers. For nonisothermal device simulations the situation is even worse. When compared with isothermal device simulation, the inclusion of temperature as a dynamic variable demands considerably more $\mathrm{CPU}$ time to find a consistent solution.

\section{THE PROGRAM TRENDY}

A few years ago, the first proposals for integrated process and device simulation were made [29], [30]. There are several arguments (see, for instance [53]) why such an integrated approach is superior to the separate handling of process and device simulation. The possibilities of the process/device simulator TRENDY go beyond the more or less "standard" device simulation capabilities of other integrated programs, since TRENDY is designed such that the implementation of almost any system of coupled flux-conservative partial differential equations is allowed.

The basic organization of the TRENDY program is shown in Fig. 1. The central data structure is situated in the center of the figure. This structure contains information about the present state of the device, such as doping concentrations, potential, carrier concentration, lattice temperature, carrier energies, etc. Moreover, the geometry of the device is an integral part of this data structure.

The shell in Fig. 1 surrounding the kernel contains application functions. These functions link the numerical algorithms and the physical models such as, for instance, carrier mobility, carrier generation, heat generation, etc. The numerical methods themselves are designed to be independent of the physical models. For example, the numerical method that solves a system of coupled continuity equations is not aware of the physical conservation law underlying the continuity equation that is presently solved. Such a strict separation between numerical methods and physical models has significant advan- tages over the conventional "integrated" approaches. It allows inclusion of user-supplied modules, while still making use of existing numerical routines and physical models.

The modules, distinguished in Fig. 1 are:

The partial differential equation solver, used to solve any
PDE:
system of flux-conservative partial differential equations. It
is the main module for device simulation.
The module for geometrical manipulations, used in the
process simulation part of TRENDY.
The module for Monte Carlo simulations of ion implantation
processes.
The module for general-purpose parameter optimization.
OPT: Intended to assist the process/device developer in tailoring
GRD: The grid manipulation module.
USR: The User Interface.

In the context of this article, a brief description of the PDE solver suffices. A special feature of our PDE solver is its flexibility. This is realized by allowing nearly any solution strategy, either coupled, decoupled (Gummel) or partly decoupled. Moreover, the calculation of the discrete fluxes and recombination terms in the PDE's is realized by using pointers to functions which can be easily set by the user.

Device simulation problems (and a part of the process simulation problems) are generally described by a system of coupled continuity equations of the following general form.

$$
\gamma \frac{\partial\left(H_{k} C_{k}\right)}{\partial t}+\lambda \nabla \mathbf{J}_{k}+R_{k}=0 \quad k=1, \cdots, N
$$

where:

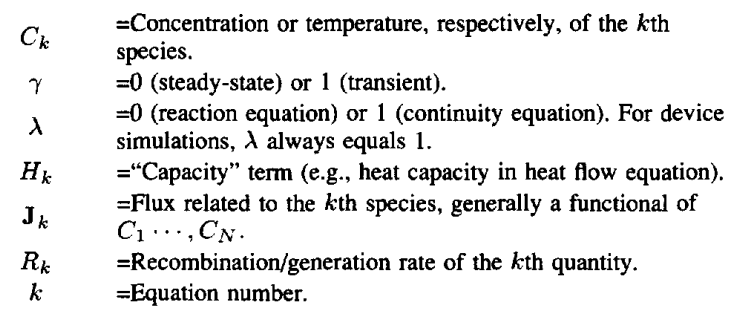

In TRENDY, the standard device equations, the energybalance equations [31], and the equations used in the thermodynamic device model (Section III), are all casted in the form of (1). After appropriate scaling of the variables, the PDE's are discretized in both space and time to obtain a (large) system of nonlinear equations. For the spatial discretization a rectangular finite difference grid is used. The time discretization is performed using a method proposed by Gear with automatic time-step selection and polynomial order selection [32], [33]. We extended this method in a straightforward way to allow for a time-dependent "capacity" term $H_{k}$ [34]. The system of nonlinear equations is solved by either a damped Newton method or a multigrid method [35]. Although the latter method promises significant (potential) advantages especially 
for large (3-D) problems, lack of generality in the restriction and prolongation operators have, at present, restricted the use to process simulation (i.e., diffusion problems) only. However, recent results from mathematics have shown strong progress in the application of multigrid methods to semiconductor device simulations [36].

The linear system resulting from a Newton iteration cycle is then solved by either a direct method or the BI-CGSTAB [37] method. The latter one is an improved version of the CGSTAB method which is gaining increasing popularity due to its smooth convergence behavior [38].

\section{THE THERMODYNAMIC MODEL}

The basic equations underlying the thermodynamic model may be deduced from a momentum expansion of Boltzmann's transport equation supplemented by an empirical ansatz for the heat flow equation [34]. A more general and physically rigorous derivation of the model equations make use of the laws of phenomenological irreversible thermodynamics applied to the composite thermodynamic system of electrons, holes and host lattice [39], [40]. Although the resulting final system of equations shows the same formal structure, the second approach avoids many disputed ambiguities in the formulation of the heat generation terms, and yields the proper dynamical equations also in the transient case. The full equation system reads

$$
\begin{aligned}
\nabla \cdot(\epsilon \nabla \psi) & =-q(p-n+N) \\
\frac{\partial n}{\partial t}-\frac{1}{q} \nabla \cdot \mathbf{J}_{n} & =-R \\
\frac{\partial p}{\partial t}+\frac{1}{q} \nabla \cdot \mathbf{J}_{p} & =-R \\
C \frac{\partial T}{\partial t}+\nabla \cdot \mathbf{S} & =H
\end{aligned}
$$

where the dependent variables are the electrostatic potential $\psi$, the electron concentration $n$, the hole concentration $p$ and the lattice temperature $T$. Here, $\epsilon, q, N, R$, and $C$ are absolute permittivity, elementary charge, net doping concentration, recombination/generation rate and heat capacity, respectively. The electron and hole current densities are given by

$$
\begin{aligned}
& \mathbf{J}_{n}=-q \mu_{n} n\left(\nabla \phi_{n}+P_{n} \nabla T\right) \\
& \mathbf{J}_{p}=-q \mu_{p} p\left(\nabla \phi_{p}+P_{p} \nabla T\right)
\end{aligned}
$$

where $\mu_{n}$ and $\mu_{p}$ denote the mobilities of electrons and holes, respectively, $P_{n}$ and $P_{p}$ are the absolute thermoelectric powers, and $\phi_{n}$ and $\phi_{p}$ are the carrier quasi-Fermi potentials. The latter are related to the state variables $(\psi, n, p, T)$ by the equations of state

$$
\begin{aligned}
& \phi_{n}=\psi-\frac{k T}{q} \ln \left(\frac{n}{n_{i e, n}}\right) \\
& \phi_{p}=\psi+\frac{k T}{q} \ln \left(\frac{p}{n_{i e, p}}\right)
\end{aligned}
$$

Here, $k$ is the Boltzmann constant, and the effective intrinsic concentrations $n_{i e, n}$ and $n_{i e, p}$ are modeled as

$$
n_{i e, n}=2 \gamma_{n}\left(\frac{2 \pi k T}{h^{2}}\right)^{3 / 2}\left(m_{n}^{*} m_{p}^{*}\right)^{3 / 4} \exp \left(-\frac{E_{g}}{2 k T}\right)
$$

$$
n_{i e, p}=2 \gamma_{p}\left(\frac{2 \pi k T}{h^{2}}\right)^{3 / 2}\left(m_{n}^{*} m_{p}^{*}\right)^{3 / 4} \exp \left(-\frac{E_{g}}{2 k T}\right)
$$

where $h$ is Planck's constant, $m_{n}^{*}$ and $m_{p}^{*}$ are the effective masses of the conduction and valence bands, respectively, $\gamma_{n}$ and $\gamma_{p}$ are the degeneracy factors [41] accounting for Fermi-Dirac statistics where the approximation by Boltzmann statistics fails, and $E_{g}$ is the effective band gap as obtained by correcting for heavy doping effects [42] or heteromaterials. One should note that all these quantities are temperaturedependent and, besides, may also be explicitly or implicitly position-dependent. This must be taken into account when the gradients of the quasi-Fermi levels in (6) and (7) are evaluated in order to express the current densities in terms of the basis variables $(\psi, n, p, T)$. The resulting relations are

$$
\begin{aligned}
\mathbf{J}_{n}= & q \mu_{n} n \mathbf{E}_{n}+q D_{n} \nabla n+q n D_{n}^{T} \nabla T \\
& -\frac{3}{2} q n D_{n} \nabla \ln \left(\frac{m_{n}^{*}}{m_{0}}\right) \\
\mathbf{J}_{p}= & q \mu_{p} p \mathbf{E}_{p}-q D_{p} \nabla p-q p D_{p}^{T} \nabla T \\
& +\frac{3}{2} q p D_{p} \nabla \ln \left(\frac{m_{p}^{*}}{m_{0}}\right)
\end{aligned}
$$

with the effective drift fields $\mathbf{E}_{n}$ and $\mathbf{E}_{p}$ defined as

$$
\begin{aligned}
\mathbf{E}_{n}= & -\nabla\left(\psi+\frac{k T}{q} \ln \gamma_{n}\right. \\
& \left.-\frac{1}{2 q} E_{g}+\frac{3}{4} \frac{k T}{q} \ln \left(\frac{m_{p}^{*}}{m_{n}^{*}}\right)\right) \\
\mathbf{E}_{p}= & -\nabla\left(\psi-\frac{k T}{q} \ln \gamma_{p}\right. \\
& \left.+\frac{1}{2 q} E_{g}+\frac{3}{4} \frac{k T}{q} \ln \left(\frac{m_{p}^{*}}{m_{n}^{*}}\right)\right)
\end{aligned}
$$

$m_{0}$ is an arbitrary mass unit, conveniently chosen as the free electron mass. The diffusion coefficients $D_{n}$ and $D_{p}$ obey the "Einstein relations"

$$
\begin{aligned}
& D_{n}=\frac{k T}{q} \mu_{n} \\
& D_{p}=\frac{k T}{q} \mu_{p}
\end{aligned}
$$

(note that this is a consequence of the constitutive relations (6) and (7) rather than a hypothesis), and the "thermal" diffusion coefficients $D_{n}^{T}$ and $D_{p}^{T}$ come out as

$$
\begin{aligned}
D_{n}^{T} & =\mu_{n}\left[\frac{k}{q}\left(\ln \left(\frac{n}{N_{c}}\right)-\frac{3}{2}\right)-P_{n}\right] \\
D_{p}^{T} & =\mu_{p}\left[\frac{k}{q}\left(\ln \left(\frac{p}{N_{v}}\right)-\frac{3}{2}\right)+P_{p}\right]
\end{aligned}
$$

where $N_{c}$ and $N_{v}$ denote the effective density of states in the conduction and the valence band, respectively. The fourth term in (12) and (13) accounts for variations in effective mass due to temperature-dependence or explicit position-dependence.

One should realize that the decomposition of the driving forces for carrier flow, according to (12) and (13), is very convenient for computational reasons, as it allows the application of a modified Scharfetter-Gummel discretization method 
(see next section). However, this decomposition is somewhat arbitrary in the sense that the first and the fourth term may also contribute to thermal diffusion through the temperaturedependence of the expressions under the $\nabla$-operator. Likewise, the degeneracy factors yield a contribution to particle diffusion when differentiating the $\ln \gamma$-terms in (14) and (15), expressing thereby the physical fact that Einstein's relations are no longer exactly valid in the case of a degenerate carrier distribution. Hence, it is somewhat imprecise and misleading to denote $D_{n}$ and $D_{p}$ as diffusion coefficients, even though this has become common practice, because the corrections are usually small.

It is also worthwhile to note that the sum of the third and fourth terms in the effective drift fields (14) and (15) may be interpreted as the gradient of the respective band edges due to both the explicit position-dependence (e.g., band gap narrowing or variation of the material type) and the temperature-dependence. The variation of the band curvatures caused by these influences is accounted for by the fourth term in (12) and (13).

From a thermodynamic point of view, the mobilities and the thermopowers have to be regarded as independent transport coefficients, which must be supplied to the modelist, either from measured data [43], [44] or from transport theory [45]. Neglecting low-temperature phenomena such as the phonondrag and other size effects, and assuming nondegenerate conditions, a practical and well-known expression for the thermoelectric power is [46]

$$
\begin{gathered}
P_{n}=-\frac{k}{q}\left(s_{n}+\frac{5}{2}-\ln \left(\frac{n}{N_{c}}\right)\right) \\
P_{p}=\frac{k}{q}\left(s_{p}+\frac{5}{2}-\ln \left(\frac{p}{N_{v}}\right)\right)
\end{gathered}
$$

where $s_{\alpha}(\alpha=n, p)$ sensitively depends on the kind of underlying electron-lattice scattering mechanism. For pure acoustic phonon scattering, we have $s_{\alpha}=-1 / 2$, while for pure ionized impurity scattering $s_{\alpha}=+3 / 2$ holds. Inserting (20) and (21) together with (16) and (17) into the relations (18) and (19) gives

$$
\begin{aligned}
& D_{n}^{T}=\frac{s_{n}+1}{T} D_{n} \\
& D_{p}^{T}=\frac{s_{p}+1}{T} D_{p}
\end{aligned}
$$

in agreement with previous work by Stratton [47]. Dorkel [48] proposed an analytical function for the numerical evaluation of $s_{\alpha}$. We simplified the calculations even further by using the following analytical function which approximates Dorkel's result to within a $1.0 \%$ RMS error:

$$
s_{\alpha}=s\left(X_{\alpha}\right)=-\frac{1}{2}+a X_{\alpha}^{b}{ }^{10} \log \left(c\left(X_{\alpha}+1\right)\right) .
$$

Here, $a, b$, and $c$ are fitting parameters having the values 1.97 , -0.039 , and 0.907 respectively, and the variable $X_{\alpha}$ is defined as

$$
X_{\alpha}=\sqrt{6 \mu_{1, \alpha} / \mu_{i, \alpha}} \quad(\alpha=n, p)
$$

with $\mu_{1, \alpha}$ and $\mu_{i, \alpha}$ denoting the acoustic phonon and ionized impurity scattering mobility of carrier type $\alpha$, respectively.
The flux $\mathbf{S}$ in (5) originates from conductive heat flow given by

$$
\mathbf{S}=-\kappa(T) \nabla T
$$

where $\kappa(T)$ is the sum of the thermal conductivities of the lattice $\kappa_{L}$, and those of the electrons and holes, $\kappa_{n}$ and $\kappa_{p}$. An a priori estimate [39] shows that $\kappa_{n}$ and $\kappa_{p}$ can be neglected in nearly all practically relevant semiconductor problems. Hence we may set $\kappa=\kappa_{L}$.

From the thermodynamic approach results an explicit expression of the heat generation term $H$ in (5). $H$ can be decomposed into a sum of three components [39]

$$
H=H_{\text {joule }}+H_{\text {rec }}+H_{\mathrm{Pt}-\mathrm{Th}}
$$

where $H_{\text {joule }}$ is the Joule heat, given by:

$$
H_{\text {joule }}=\frac{\mathbf{J}_{n}^{2}}{q \mu_{n} n}+\frac{\mathbf{J}_{p}^{2}}{q \mu_{p} p},
$$

$H_{\text {rec }}$ is the recombination heat

$$
H_{\mathrm{rec}}=q R\left(\phi_{p}-\phi_{n}+T\left(P_{p}-P_{n}\right)\right),
$$

and $H_{\mathrm{Pt}-\mathrm{Th}}$ includes Peltier and Thomson heat

$$
H_{\mathrm{Pt}-\mathrm{Th}}=-T\left(\mathbf{J}_{n} \cdot \nabla P_{n}+\mathbf{J}_{p} \cdot \nabla P_{p}\right) .
$$

The latter expression gives a contribution whenever an electric current traverses a gradient of the thermopowers. This may occur either due to local variations of temperature $\left(\nabla P_{c}=\left(\partial P_{c} / \partial T\right) \nabla T\right.$, Thomson effect $)$, or due to rapidly varying carrier concentrations $\left(\nabla P_{n}=\left(\partial P_{n} / \partial n\right) \nabla n, \nabla P_{p}=\right.$ $\left(\partial P_{p} / \partial p\right) \nabla p$, Peltier effect along pn-junctions), or due to discontinuous changes of the thermopowers along material interfaces (classical Peltier effect).

It has been disputed whether or not it is adequate to denote (28) as Joule heat. An alternative suggestion would be to define the Joule heat in a semiconductor in analogy to the situation in a metal; that is as the inner product of the electric field $\mathbf{E}$ and the total current $\mathbf{J}$. In our opinion, this is just a matter of terminology. Joule heat was defined in the late 19th Century, when no difference was made between electron and hole conduction. A "natural" way to include the Joule heat in the total heat generation $H$ is using (28). If, instead, somebody prefers to stick at the definition $\mathbf{J} \cdot \mathbf{E}$, then one still has to assign a proper name to the difference between (28) and $\mathbf{J} \cdot \mathbf{E}$ :

$$
\frac{\mathbf{J}_{n}^{2}}{q \mu_{n} n}+\frac{\mathbf{J}_{p}^{2}}{q \mu_{p} p}-\mathbf{J} \cdot \mathbf{E}
$$

We feel that such an artificial splitting up of terms is not very helpful for the intuitive understanding of the physical content of (27).

The expression (29) of the recombination heat $H_{\mathrm{rec}}$ is, strictly speaking, only valid under steady-state conditions [39]. In practice, however, as long as the carrier concentrations show a quasi-stationary behavior on the time scale typical of thermal transients, and provided that we do not deal with very fast switching times ( $\leq 10 \mathrm{~ns}),(29)$ can still be used in transient problems without introducing a significant error. 
The relative weight of the individual contributions (28)-(30) to the total heat generation, strongly depends on the device type considered and the respective operating conditions. In the simulations discussed in Section V of this paper, we encountered only situations where Joule heating was predominant whenever a significant temperature increase occurred.

\section{IMPLEMENTATION ASPECTS}

A general remark on the numerical implementation of the thermodynamic model concerns the hierarchy of time constants. As already mentioned, electrical transients are usually much faster than thermal transients, and this fact makes it impractical to simulate both electrical and thermal behavior in transient mode. Instead, it is, in most cases, sufficient to simulate thermal transients when the electrical state variables have already settled to steady-state conditions. Only at very short pulse rise times ( $<10 \mathrm{~ns}$ typically) this simplified approach might fail, because here the contribution to heat generation caused by electrical transient effects may largely exceed Joule heating [39]. However, even in this case the overall influence on the device temperature resulting from the short period of electrical transient behavior turns out to be small in most applications and, hence, may be neglected using the quasisteady state approach. Therefore, although TRENDY features, due to its flexible structure, the possibility of simulating a device in fully transient mode (i.e., $n, p$, and $T$ ), it is usually more practical to simulate only the lattice temperature $T$ in the transient model.

For the "standard" device equations, two schemes for the solution of the nonlinear equations resulting from the discretization of the PDE's are generally in use: the decoupled (Gummel) and the fully coupled approach. In the case of the four dependent variables used here, an alternative approach is more convenient.

The structure of (12) and (13), as well as the complicated form of the heat generation model $(27)-(30)$ indicate that a coupled solution of the variables $\psi, n, p$, and $T$ will give a rather complex problem to solve. Moreover, since the total number of (nonsparse) elements in the Jacobian matrix is quadratically proportional to the number of solution variables, the problem becomes extremely large for 2-D simulations. A natural choice is to partly decouple the solution variables and follow the solution strategy as outlined in Fig. 2.

With this method, first the subsystem for $(\psi, n, p)$ is solved in a coupled solution mode using an initial temperature $T_{0}$. Thereafter, the equation for $T$ is solved, using the calculated values of $\psi, n$, and $p$. This results in a certain temperature distribution $T$, to be used for a new solution of the system $(\psi, n, p)$. The process is repeated until self-consistence is attained.

A natural ansatz for the discretization of the current transport equations is to add the thermal diffusion component to the drift component. Both components can be expected to fall within a "reasonable" range compared to the carrier diffusion term. So $\mathbf{J}_{n}$ and $\mathbf{J}_{p}$ can be rewritten as

$$
\begin{aligned}
& \mathbf{J}_{n}=q \mu_{n} n\left(\mathbf{E}_{n}+\rho_{n} \nabla T\right)+q D_{n} \nabla n \\
& \mathbf{J}_{p}=q \mu_{p} p\left(\mathbf{E}_{p}-\rho_{p} \nabla T\right)-q D_{p} \nabla p
\end{aligned}
$$

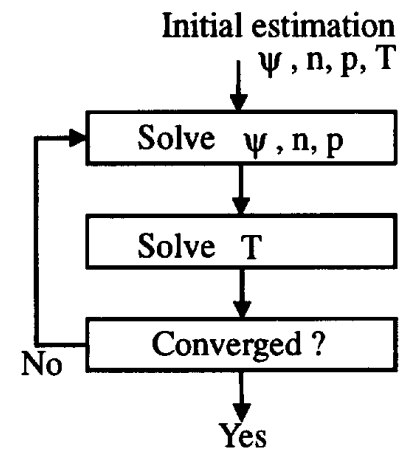

Fig. 2. Solution process for the nonisothermal device equations.

where:

$$
\rho_{\alpha}=U_{t} \frac{s_{\alpha}+1}{T}=\frac{k}{q}\left(s_{\alpha}+1\right) \quad(\alpha=n, p) .
$$

Note that in the present stage of implementation variations in the effective masses have been neglected. As both $\mathbf{E}_{n} / \mathbf{E}_{p}$ and $\rho_{\alpha} \nabla T$ are not expected to change rapidly from node $i$ to node $i+1$, the discretization of (32) and (33) may be properly performed by a "standard" Scharfetter-Gummel scheme [49] with a modified $\mathbf{E}$ term. For the isothermal case, the interpolation formulae reduce to the original Scharfetter-Gummel expressions.

The discretization of the heat-flux equation (26) is trivial as long as the thermal conductivity $\kappa(T)$ is independent of temperature, which is a very close approximation for most metals. Silicon and silicon oxide, however, show a pronounced temperature dependence in $\kappa(T)$ [50], which can be described by the function:

$$
\kappa=K_{0} T^{-\alpha}
$$

where $K_{0}$ and $\alpha$ are material-dependent constants, $\mathbf{S}$ can now be rewritten as:

$$
\mathbf{S}=K_{0} T^{-\alpha} \nabla T
$$

or

$$
\mathbf{S}=\frac{1}{1-\alpha} K_{0} \nabla\left(T^{1-\alpha}\right) .
$$

The advantage of using (37) instead of (36) is that for $C=0$ (5) becomes linear in $T^{1-\alpha}$. Thus better convergence properties are to be expected. Discretization of $\mathbf{S}$ is now straightforward in the interior of the individual materials each of which shows a temperature dependence of the thermal conductivity, as given by (35).

For the electric fluxes, the usual (Dirichlet and Neumann) boundary conditions apply.

To avoid "blowing up" the device when solving the heatflow equation in steady-state, the device should be attached to a heat sink somewhere along the boundary, or finite heat conduction through the surface should be allowed for.

A heat sink is modeled by a simple Dirichlet boundary condition:

$$
T=T_{0}
$$




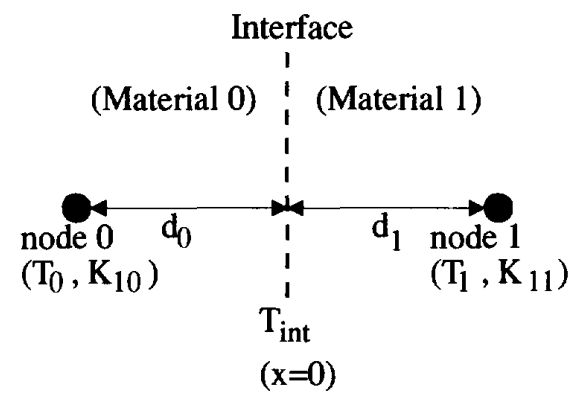

Fig. 3. Illustration of the discretization process.

Surface heat transfer (going outside the domain) is modeled using an effective thermal resistance, with the following condition applied:

$$
\mathbf{S} \cdot \mathbf{n}=\left(T-T_{0}\right) / k_{0}
$$

where $k_{0}$ is the distributed thermal resistance in $\mathrm{Kcm}^{2} \mathrm{~W}^{-1}$, and $\mathbf{n}$ is the outward unit normal. Condition (39) prescribes the heat flux $\mathbf{S}$ at the boundary. With the concept of thermal resistance, one can also model the convective heat transfer along the surface of the device and the ambient air. In this case one generally speaks of a "heat transfer coefficient" which is the inverse of the thermal resistance $k_{0}$. This coefficient depends on many factors, notably the composition of the air and the kind of gas motion past the surface. Numerical values for various situations may be found in [51], for instance.

Along symmetry lines and at places where heat conduction is negligible, homogeneous Neumann boundary conditions apply

$$
\mathbf{S} \cdot \mathbf{n}=0
$$

(i.e., the projection of the energy flow on the interface normal is zero).

In the transient mode it is often a good approximation to use Neumann boundary conditions exclusively as long as the reflections of the "heat front" on the boundary do not introduce perceptible perturbations in the device simulation.

The treatment of interfaces between adjacent materials which have different temperature-dependences in the thermal conductivities is a rather difficult issue. This is caused by the fact that, with an explicit boundary representation as used in TRENDY, the temperature along an interface is not contained in the set of solution variables and, therefore, the interface flux $\mathbf{S}_{\text {int }}$ is difficult to calculate. This quantity, however, is needed to express the continuity of heat flux across material interfaces as a boundary condition. Fig. 3 illustrates the discretization process. In the case of two materials having constant thermal conductivities, the interface flux is calculated just as it would be done for the dielectric displacement flux:

$$
\mathbf{S}_{\mathrm{int}}=\frac{T_{1}-T_{0}}{\frac{d_{1}}{K_{1}}+\frac{d_{0}}{K_{0}}} .
$$

Thus, the interface flux $S_{\text {int }}$ is given in an explicit form. In the case of different temperature dependencies in the thermal conductivities, no explicit expression can be found for the general case. Instead, an implicit equation must be solved numerically. The procedure is as follows.

Since it is assumed that the energy flux is continuous along interfaces it follows that:

$$
\left.K_{10} \frac{\partial T}{\partial x}\right|_{x \uparrow 0}=\left.K_{11} \frac{\partial T}{\partial x}\right|_{x \downarrow 0} .
$$

Assuming that material 0 has a thermal conductivity $K_{c 0} T^{-\alpha_{0}}$ and material 1 has $K_{\mathrm{c} 1} T^{-\alpha_{1}}$ it follows:

$$
\left.\frac{K_{c 0}}{1-\alpha_{0}} \frac{\partial\left(T^{1-\alpha_{0}}\right)}{\partial x}\right|_{x \uparrow 0}=\left.\frac{K_{c 1}}{1-\alpha_{1}} \frac{\partial\left(T^{1-\alpha_{1}}\right)}{\partial x}\right|_{x \downarrow 0} .
$$

Now, the partial derivatives in (43) are expressed by finite differences:

$$
\begin{aligned}
\frac{K_{c 0}}{1-\alpha_{0}} & \frac{T_{\mathrm{int}}^{\left(1-\alpha_{0}\right)}-T_{0}^{\left(1-\alpha_{0}\right)}}{d_{0}} \\
= & \frac{K_{c 1}}{1-\alpha_{1}} \frac{T_{\mathrm{int}}^{\left(1-\alpha_{1}\right)}-T_{1}^{\left(1-\alpha_{1}\right)}}{d_{1}},
\end{aligned}
$$

which is an equation of the form:

$$
C_{0} T_{\mathrm{int}}^{\left(1-\alpha_{0}\right)}+C_{1} T_{\mathrm{int}}^{\left(1-\alpha_{1}\right)}=C_{2} .
$$

Since this equation is not analytically solvable in the general case, the interface temperature $T_{\text {int }}$ has to be calculated by a root-finding procedure using algebraic derivatives (e.g., Newton-Raphson). Then the discrete heat flux $\mathbf{S}_{\text {int }}$ through the interfacial layer is given by:

$$
\mathbf{S}_{\text {int }}=\frac{K_{c 0}}{1-\alpha_{0}} \frac{T_{\text {int }}^{\left(1-\alpha_{0}\right)}-T_{0}^{\left(1-\alpha_{0}\right)}}{d_{0}} .
$$

If $\alpha_{0}$ and $\alpha_{1}$ are equal, (44) can be solved explicitly. After some algebra it follows $\left(\alpha=\alpha_{0}=\alpha_{1}\right)$ :

$$
T_{\text {int }}=\left(\frac{K_{c 0} d_{1}\left(T_{0}\right)^{1-\alpha}+K_{c 1} d_{0}\left(T_{1}\right)^{1-\alpha}}{K_{\mathrm{c} 0} d_{1}+K_{\mathrm{c} 1} d_{0}}\right)^{1 / 1-\alpha}
$$

and again (46) can be used for the calculation of the interface flux.

Physically unrealistic thermal effects can originate from the assumption of ideal ohmic contact boundaries on a semiconducting material. At such boundary segments, it is common practice to postulate thermal equilibrium and space charge neutrality in the derivation of the boundary conditions [52] Therefore, as long as the minority carrier concentration has not settled to its equilibrium value, a discontinuity in the minority carrier distribution will occur underneath the contacted surface area. As a consequence, the electric conductivity of the minority carriers makes a sudden jump towards a low value and, thereby, the Joule heat generation becomes extremely high. This phenomenon is an artefact which reflects the physical inconsistencies introduced by the contradictory assumptions of thermal equilibrium and simultaneous heat flow. Moreover, the convergence speed of the simulation is sensitively deteriorated. We found a solution to this problem by introducing a thin layer of an artificial material with high recombination velocity as a "buffer" between ohmic contact and semiconductor. Thus finite volume recombination replaces 


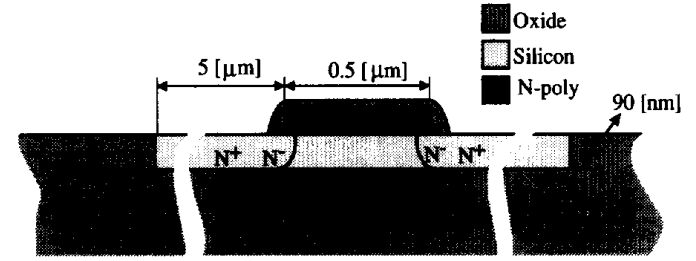

Fig. 4. Geometry of the $0.5 \mu \mathrm{m}$ SOI device.

the surface recombination at the silicon-metal contact. Such an approach yields not only a physically sound solution, but also prevents numerical ill-performance. In TRENDY, this artificial material is also used as a buffer between current-controlled contacts and silicon, saving the pentadiagonal structure of the Jacobian matrix which results from a Newton iterate during the solution of the nonlinear system of equations [34].

\section{AN EXAMPLE: EleCtro-THERMAL SIMULATION OF AN SOI MOSFET}

In MOSFET's fabricated by means of Silicon on Insulator (SOI) technology, a considerable temperature increase is found [21] when operated at high gate and drain voltages. This temperature increase has a pronounced effect on the output characteristics of such SOI devices since the $\mathrm{V}_{\mathrm{ds}}-\mathrm{I}_{\mathrm{ds}}$ curves exhibit a characteristic negative slope at high drain and gate voltages. As a result of our simulations, we will demonstrate that such a negative slope is caused by the temperature dependence of the carrier saturation velocity. Moreover, it will be shown that in the case of avalanche generation, inclusion of temperature dependence in the impact ionization models is an absolute necessity for accurate nonisothermal simulations.

The "floating bulk" SOI device investigated is shown in Fig. 4. The width (in the direction perpendicular to the simulation plane) is $8 \mu \mathrm{m}$. The device itself is completely embedded in a buried oxide layer with a thickness of about $0.4 \mu \mathrm{m}$. Based on process data, the doping profiles of this device have been simulated using the process simulation part of TRENDY [53].

Nonisothermal device simulations have been performed. The total heat generation rate at a bias of $\mathrm{V}_{\mathrm{ds}}=5 \mathrm{~V}$ and $\mathrm{V}_{\mathrm{gs}}=4 \mathrm{~V}$ is shown in Fig. 5. The largest heat generation term is the Joule heat. Recombination heat generation occurs only close to the drain where the onset of impact ionization can be observed. Recombination heating is about four orders of magnitude smaller than Joule heating. The Thomson-Peltier heat generation occurs at locations where large gradients in the thermo-electric powers $P_{n}$ and $P_{p}$ occur. This is near the source and drain where large gradients in carrier concentration result in large gradients of $P_{n}$ and $P_{p}$. The Thomson-Peltier heat generation is about one order of magnitude smaller than the Joule heat generation and is therefore of only minor influence.

The internal temperature distribution in the SOI device, at the given bias conditions, is shown in Fig. 6 . The active device region is concentrated in a very small area around the peak temperature $(500 \mathrm{~K})$. The maximum device temperature at several bias points is shown in Fig. 7. These temperatures are

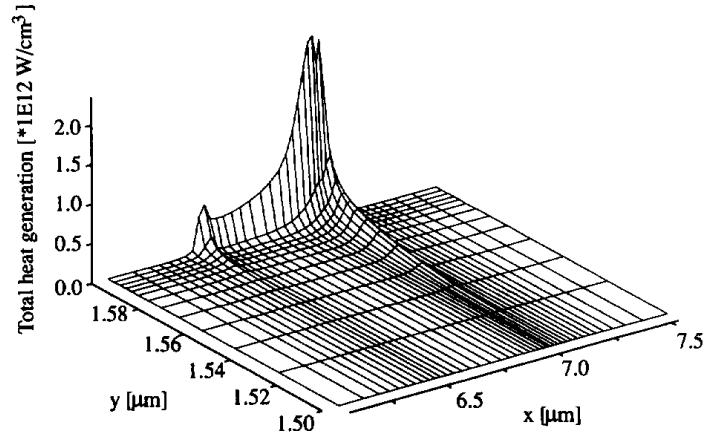

Fig. 5. Total heat generation at $V_{d s}=5 \mathrm{~V}, V_{g s}=4 \mathrm{~V}$.

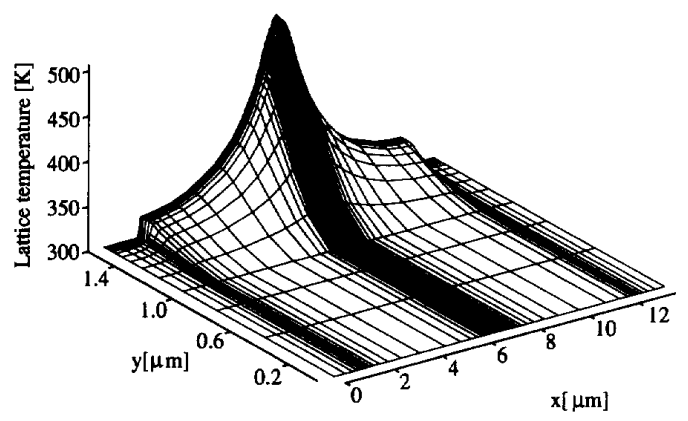

Fig. 6. Internal temperature distribution at $V_{d s}=5 \mathrm{~V}, V_{g s}=4 \mathrm{~V}$.

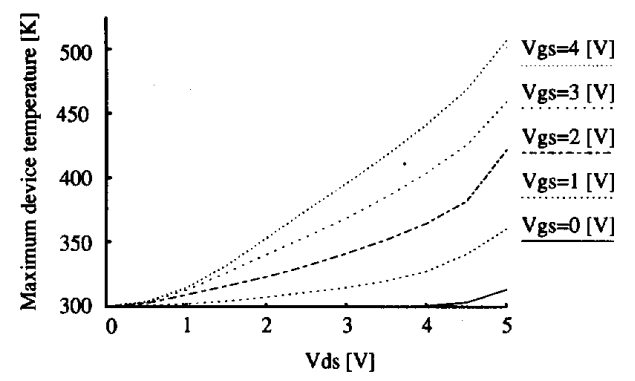

Fig. 7. Maximum device temperature at several bias points.

close to the temperature predictions obtained by extrapolation of the measurements by Lifka et al. [21].

The simulated nonisothermal $\mathrm{V}_{\mathrm{ds}}-\mathrm{I}_{\mathrm{ds}}$ characteristics are shown in Fig. 8, together with the measured characteristics (drawn lines). The measured curves are scaled in the $y$ direction to correct for process and device simulation inaccuracies. As mentioned in Section I, accurate and reliable 2-D process simulations are difficult to obtain, particularly for submicron devices. The inaccuracies found here can be attributed to the fact that the physical parameters necessary for accurate process and device simulation were not determined for this SOI process in its research state. By following the scaling approach, we are able to compare the characteristic shape of the $V_{d s}-I_{d s}$ curves from the measurements with those from the simulations. The curves show a good agreement; particularly the negative slope at high gate and drain voltages and the "kink" effect at low gate voltages are clearly visible. 


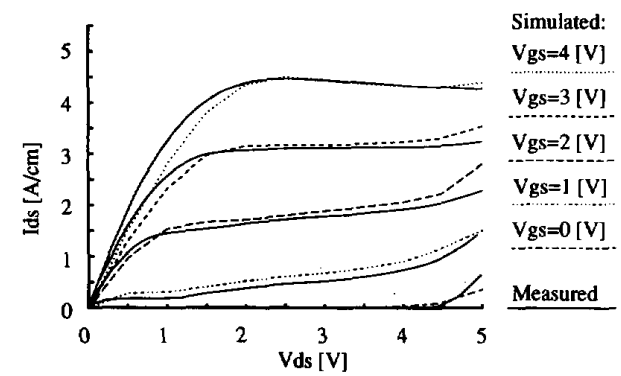

Fig. 8. Simulated nonisothermal and measured $V_{d s}-I_{d s}$ characteristics.

TABLE I

IMPACT IONIZATION CONSTANTS

\begin{tabular}{c|ccc}
\hline & $a_{0}\left[\mathrm{~cm}^{-1}\right]$ & $b_{0}\left[\mathrm{~V} \mathrm{~cm}{ }^{-1}\right]$ & $\beta\left[\mathrm{K}^{-1}\right]$ \\
\hline electrons & $6.2 \mathrm{e} 5$ & $1.08 \mathrm{e} 6$ & $1.2 \mathrm{e}-3$ \\
holes & $2.0 \mathrm{e} 6$ & $1.98 \mathrm{e} 6$ & $5.6 \mathrm{e}-4$ \\
\hline
\end{tabular}

Compared to the isothermal characteristics, it is found that device heating leads to lower currents at higher temperatures. Our simulations revealed that this effect is almost completely caused by the temperature dependence of the carrier saturation velocity, which has been modeled in TRENDY by:

$$
V_{\text {sat }}=V_{\text {sat } 0}\left(\frac{T}{T_{0}}\right)^{-\gamma}
$$

where $\gamma$ takes the theoretical value of 0.5 [54].

Other thermal effects observed in our simulations are a less-pronounced kink-effect and a perceptible reduction of the avalanche current. The latter is caused by the temperature dependence of the impact ionization model. Using Grant's avalanche model [55], the impact ionization rate $\alpha$ is calculated as:

$$
\begin{aligned}
\alpha & =a_{0} \exp \left[-\left(\frac{b}{F}\right)\right] \\
b & =b_{0}(1+\beta(T-300))
\end{aligned}
$$

where $a_{0}, b_{0}$, and $\beta$ are constants given in Table I. $F$ is the electric field along the direction of the current flow and $T$ is the temperature in Kelvin.

If no temperature dependence is allowed for in the avalanche model (as is the case in most simulation programs), a breakdown is predicted due to the positive feedback of avalanche multiplication. For comparison, Fig. 9 shows a simulation without temperature dependence of impact ionization.

\section{SUMMARY AND CONCLUSIONS}

In this paper, we have reported the incorporation of nonisothermal effects in the 2-D numerical process/device simulator TRENDY. The thermodynamic system of equations is solved using a partly decoupled method. The current transport equations have been rewritten such that a "standard" Scharfetter-Gummel discretization scheme can be used. Furthermore, a boundary discretization method has been proposed which permits us to solve the heat flow equation for a wide range of materials.

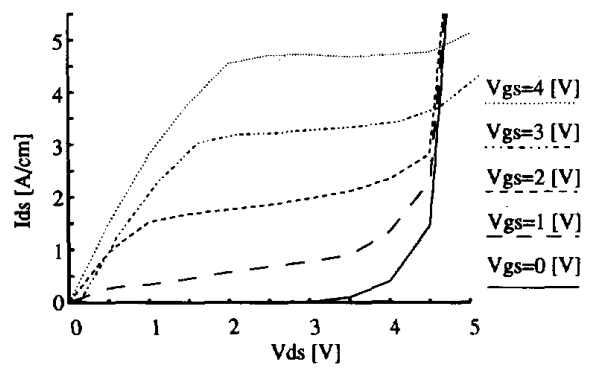

Fig. 9. Breakdown effect if avalanche model is temperature independent.

Using TRENDY, the electro-thermal behavior of an uitrathin SOI MOSFET has been simulated. It shows that the simulation of the electrical behavior alone is no longer sufficient for these kind of MOSFET's. It has been demonstrated that the negative slope in the $\mathrm{V}_{\mathrm{ds}}-\mathrm{I}_{\mathrm{ds}}$ characteristics at higher gate voltages is caused by the temperature-dependence of the carrier saturation velocity. For an accurate analysis it is also required to include temperature dependence in the impact ionization model.

Future work at the MESA institute concerning process/device simulation will concentrate on thermomagnetic effects in semiconductors, influence of radiation, simulation of electromigration phenomena and other kinds of applications of the TRENDY program.

\section{ACKNOWLEDGMENT}

The authors would like to thank Frans Klaassen and Pierre Woerlee from Philips Research Labs, Eindhoven, for the helpful discussions and the SOI MOSFET data. Paul de Zeeuw from the Centre for Mathematics and Informatics, Amsterdam for his support in implementing the BI-CGSTAB method in TRENDY.

\section{REFERENCES}

[1] S. P. Gaur and D. H. Navon, "Two-dimensional carrier flow in a transistor structure under nonisothermal conditions," IEEE Trans. Electron Devices, vol. ED-23, pp. 50-57, 1976.

[2] P. Walker, "Electrical and thermal modeling of power semiconductor devices using numerical methods," Ph.D. dissertation, University of Liverpool, United Kingdom, 1988.

[3] P. A. Gough, P. Walker, and K. R. Whight, "Electrothermal simulation of power semiconductor devices," in Proc. 3rd Int. Symp. on Power Semiconductor Devices and IC's (ISPSD '91), Baltimore, MD, 1991, pp. 89-94.

[4] P. Dickinger, G. Nanz, and S. Selberherr, "Self-consistent simulation of heat generation and conduction in semiconductor devices," in Proc. 18th Yugoslav Conf. Microelectronics MIEL-90, Ljubljana, Yugoslavia, 1990, pp. 157-160.

[5] P. Ciampolini, A. Pierantoni, and G. Baccarani, "Three-dimensional, self-consistent modeling of MOS devices under nonisothermal regime," in Simulation of Semiconductor Devices and Processes, vol. 4, W. Fichtner and D. Aemmes, eds. Konstanz, Germany: Hartung-Gorre Verlag, 1991, pp. 149-156.

[6] K. Kells, S. Müller, W. Fichtner, and G. Wachutka, "Simulating temperature effects in multi-dimensional silicon devices with generalized boundary conditions," in Simulation of Semiconductor Devices and Processes, vol. 4, W. Fichtner and D. Aemmes, eds. Konstanz, Germany: Hartung-Gorre Verlag, 1991, pp. 141-148.

[7] K. Mayaram, J. H. Chern, L. Arledge, and P. Yang, "Electrothermal simulation tools for analysis and design of ESD protection devices," in IEDM Tech. Dig, Washington, DC, 1991, pp. 909-912. 
[8] W. Nakwaski, "Thermal properties of the Burrus-type light-emitting diode," IEEE Trans. Electron Devices, vol. ED-33, pp. 889-907, 1986.

[9] D. K. Sharma and K. V. Ramanathan, "Modeling thermal effects on MOS I-V characteristics," IEEE Electron Dev. Lett, vol. EDL-4, PP. 362-364, 1983.

[10] M. S. Adler, "Accurate calculations of the forward drop and power dissipation in thyristors," IEEE Trans. Electron Devices, vol. ED-25, pp. 16-22, 1978

[11] A. Chryssafis and W. Love, "A computer-aided analysis of onedimensional thermal transients in n-p-n power transistors," Solid-State Electronics, vol. 22 , pp. 249-256, 1979

[12] N. Kusnezov and J. Smith, "Modeling of EOS in silicon devices," in Proc. EOS/ESD Symp., Sept. 1979, pp. 132-138.

[13] A. Schütz, S. Selberherr, and H. W. Pötzl, "Temperature distribution and power dissipation in MOSFET's," Solid-State Electronics, vol. 27 no. 4, pp. 394-395, 1984.

[14] A. Nakagawa and H. Ohashi, "A study of GTO tum-off failure mechanisms," IEEE Trans. Electron Devices, vol. ED-31, pp. 273-279, 1984

[15] A. Nakagawa and D. H. Navon, "A time- and temperature-dependen 2-D simulation of the GTO thyristor turn-off process," IEEE Trans. Electron Devices, vol. ED-31, pp. 1156-1163, 1984.

[16] A Nakagawa, "A time- and temperature-dependent two-dimensional simulation of the GTO thyristor turn-off process, II-Inductive load case," Solid-State Electronics, vol, 28, pp. 677-687, 1985.

[17] H. Fukui and T. Yaginuma, "Two-dimensional numerical analysis of the turn-off process in a GTO under inductive load," IEEE Trans. Electron Devices, vol. ED-32, pp. 1830-1834, 1985.

[18] A. Nathan, W. Allegretto, K. Chau, and H. P. Baltes, "Electrical and thermal analysis of VLSI contacts and vias," in Simulation of Semiconductor Devices and Processes (SISDEP), vol. 3. Bologna, Italy: Tecnoprint, 1988, pp. 589-598.

[19] P. G. Mautry and J. Trager, "Self-heating and temperature measurement in sub- $\mu \mathrm{m}$ MOSFET's," in ESSDERC 89 Tech. Dig., 1989, pp. 675-678.

[20] N. Haik, D. Gat, R. Sadon, and Y. Nissan-Cohen, "Measurement of transient heating in a $1.1 \mu \mathrm{m}$ PMOSFET using thermal imaging," IEEE Electron Device Letters, vol. EDL-12, pp. 611-613, 1991.

[21] H. Lifka and P. H. Woerlee, "Thin SIMOX SOI material for half-micron CMOS," in ESSDERC 90 Tech. Dig., 1990, pp. 453-456.

[22] S. Beeck et al., "Investigations of GaAs/AlGaAs quantum well lasers by micro raman spectroscopy," in ESSDERC-89 Tech. Dig., 1989.

[23] H. Brugge and P. W. Epperlein, "Mapping of local temperatures on mirrors of GaAs/AlGaAs laser diodes," Appl. Phys. Lett., vol. 56, pp. 1049-1051, 1990.

[24] A. Nathan, H. Baltes, and W. Allegretto, "Review of physical models for numerical simulation of semiconductor sensors," IEEE Trans. Electron Devices, vol. 9, pp. 1198-1208, 1990

[25] A. W. van Herwaarden and P. M. Sarro, "Thermal sensors based on the Seebeck effect," Sensors and Actuators vol. 10, pp. 321-346, 1986.

[26] U. Gerlach-Meyer, "Asymmetric Joule heat production at a point contact," Appl. Phys., vol. A33, pp. 161-165, 1984.

[27] H. Baltes, D. Moser, R. Lenggenhager, O. Brand, and G. Wachutka, "Thermomechanical microtransducers by CMOS technology combined with micromachining," in Microsystem Technologies 91, R. Krahn and H. Reichl, eds. Berlin, Germany: VDE-Verlag, 1991, pp. 98-103.

[28] S. Rudin, G. Wachutka, and H. Baltes, "Thermal effects in magnetic microsensor modeling," Sensors and Actuators, vol. A25-27, pp. 731-735, 1991.

[29] D. Collard, B. Baccus, and E. Dubois, "IMPACT 1-2-3, an integrated 2-D process/device simulator for M.O.S. technology," in Software Tools for Process, Device and Circuit Modeling. W. Crans, ed. Dublin, Ireland: Boole Press, 1989, pp. 16-31.

[30] A. Gérodolle, C. Corbex, A. Poncet, T. Pédron, and S. Martin, "TITAN 5. A two-dimensional process and device simulator," in Software Tools for Process, Device and Circuit Modeling, W. Crans, ed. Dublin, Ireland: Boole Press, 1989 , pp. 56-67.

[31] K. Bґo tekjaer, "Transport equations for electrons in two-valley semiconductors," IEEE Trans. Electron Devices, vol. ED-17, pp. 38-47, 1970 .

[32] C. W. Gear, "The automatic integration of stiff ordinary differential equations," in Information Processing 68. Amsterdam: North-Holland 1969.

[33] C. W. Gear, "The automatic integration of ordinary differential equations," Communications of the ACM, vol. 14, no. 3, pp. 176-179, 1971

[34] P. B. M. Wolbert, "Modeling and simulation of semiconductor devices in TRENDY: Electrical, thermal and hydrodynamic behavior," Ph.D dissertation, University of Twente, Enschede, The Netherlands, 1991.

[35] K. Brand, M. Lenke, and J. Linden, "Multigrid bibliography," in Multigrid Methods, Frontiers in Applied Mathematics, vol. 3, S. F. McCormick, ed. Philadelphia, PA: SIAM, 1987.
[36] J. Molenaar, "Multigrid methods semiconductor device simulation," Ph.D. dissertation, Centre for Mathematics and Computer Science, Amsterdam, The Netherlands, 1992.

[37] H. A. van der Vorst, "BI-CGSTAB: A fast and smoothly converging variant of BI-CG for the solution of nonsymmetric linear systems," SIAM J. Sci. Statist. Comput., vol. 13, no. 2, 1992.

[38] G. Heiser, C. Pommerel, J. Weiss, and W. Fichtner, "Three-dimensional numerical semiconductor device simulation; Algorithms, architectures, results," IEEE Trans. Computer-Aided Design, vol. 10, pp. 1218-1230, 1991.

[39] G. K. Wachutka, "Rigorous thermodynamic treatment of heat generation and conduction in semiconductor device modeling,". IEEE Trans. Computer-Aided Design, vol. 9, pp. 1141-1149, 1990.

[40] G. K. Wachutka, "Unified framework for thermal, electrical, magnetic, and optical semiconductor device modeling," COMPEL, vol. 10, pp. 311-322, 1991

[41] Z. Yu and R. W. Dutton, "SEDAN-III-A generalized electronic material device analysis program," Tech. Rep., Stanford Electronics Lab, Stanford Univ., July 1985

[42] J. W. Slotboom and H. C. de Graaff, "Bandgap narrowing in silicon bipolar transistors," IEEE Trans. Electron Devices, vol. ED-24, pp. $1123-1125,1977$.

[43] T. H. Geballe and G. W. Hull, "Seebeck effect in silicon," Phys. Rev. vol. 98 , pp. 940-947, 1955.

[44] M. E. Brinson and W. Dunstan, "Thermal conductivity and thermoelectric power of heavily doped n-type silicon," J. Phys., vol. C3, pp. 483-491, 1970.

[45] C. Herring, "Theory of the thermoelectric power of semiconductors," Phys. Rev., vol. 96, pp. 1163-1187, 1954.

[46] K. Seeger, Semiconductor Physics. Berlin, Germany: Springer Verlag, 1982 , p. 81

[47] R. Stratton, "Semiconductor current-flow equations (diffusion and degeneracy)," IEEE Trans. Electron Devices, vol. ED-19, pp. 1288-1292, 1972.

[48] J. M. Dorkel, "On electrical transport in nonisothermal semiconductors," Solid-State Electronics, vol. 6, pp. 819-821, 1983.

[49] D. Scharfetter and H. K. Gummel, "Large-signal analysis of a silicon read diode oscillator," IEEE Trans. Electron Devices, vol. ED-16, pp. 64-77, 1969.

[50] C. J. Glassbrenner and G. A. Slack, "Thermal conductivity of silicon and germanium from $3 \mathrm{~K}$ to melting point," Phys. Rev., vol. 134, no. 4A, pp. 1058-1069, 1964.

[51] A. J. Chapman, Fundamentals of Heat Transfer. New York: MacMillan, 1987.

[52] S. Selberherr, Analysis and Simulation of Semiconductor Devices. Wien, Austria: Springer-Verlag, 1984.

[53] E. van Schie, "TRENDY: An integrated program for IC process and device simulation," Ph.D. dissertation, Univ. of Twente, Enschede, The Netherlands, 1990.

[54] P. S. Kireev, Semiconductor Physics. Moscow, Russia: MIR Publishers, 1978

[55] W. N. Grant, "Electron and hole ionization rates in epitaxial silicon at high electric fields," Solid-State Electronics, vol. 16, no. 10, pp. 1189-1203, 1973

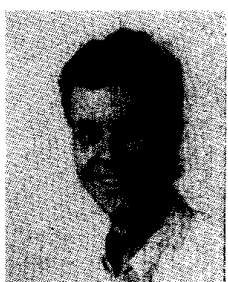

Philip B. M. Wolbert (S'90-M'91) received the M.Sc. and the Ph.D. degrees in electrical engineering from the University of Twente, Enschede, The Netherlands, in 1986 and 1991, respectively.

His Ph.D. dissertation dealt with the modeling and numerical simulation of semiconductor devices, with the emphasis on thermodynamic and hydrodynamic device behavior. $\mathrm{He}$ is one of the originators of the TRENDY program, an integrated 2-D process/device simulator. Since early 1992 he has worked on several reliability aspects of submicron CMOS processes within the MOS3 facility of Philips Semiconductors Nijmegen, The Netherlands. He also represents Philips in the European JESSI program "Special Tools and Methods for High Reliability." He is a consultant for PROSA, a company which specializes on scientific software and third party research. His research interests include semiconductor device modeling. IC reliability simulation, latch-up modeling and parameter extraction. 


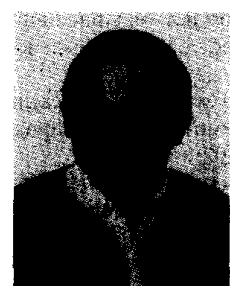

Gerhard K. M. Wachutka (M'90) received the D.Sc. degree from the Ludwig-MaximiliansUniversität Munich in 1985.

From 1985 to 1988 , he was with Siemens Corporate Research \& Development, Munich, where he headed a modeling group active in the development of modern high power semiconductor devices. In 1989 he joined the Fritz-Haber-Institute of the Max-Planck-Society in Berlin working in the field of theoretical solid-state physics. Since 1990 he is heading the microtransducers modeling and characterization group of the Physical Electronics Laboratory at the ETH Zurich. He is also leading the microtransducers modeling module of the Swis Federal Priority Program $M^{2} S^{2}$ (Micromechanics on Silicon in Switzerland).

Dr. Wachutka is member of the American Electrochemical Society and the German Physical Society.

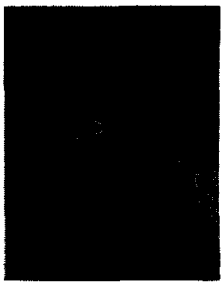

Benno H. Krabbenborg received the M.Sc. degree in electrical engineering from the University of Twente, The Netherlands in 1990. In the same year he joined the IC-Technology and Electronics group at the University of Twente where he is working towards his Ph.D. degree.

His Ph.D. research deals with modeling of coupled thermal-electrical (breakdown-) processes in bipolar transistors. Therefore his interests include 2-D nonisothermal device simulation, and 3-D thermal-electrical simulation using lumped circuit models.

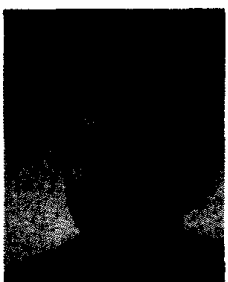

Ton J. Mouthaan (M'89) received the B.Sc. degree in electrical engineering in 1973, and the M.Sc. degree in 1977, both from the University of Twente, Enschede, The Netherlands. In 1986, he received the $\mathrm{Ph} . \mathrm{D}$. degree from the same university.

After a short period as Research Assistant at the University of Twente, he went as a Lecturer to the University of Zambia to establish a Department of Electronics there. His Ph.D. research (at the University of Twente) dealt with high energy ion implantation for the fabrication of vertical devices. After two more years at University of Zambia, as senior Lecturer, he returned to the University of Twente as Associate Professor heading research groups in modeling and simulation and CAD for the synthesis of analog circuits. He also lectures on IC Technology, and is currently member of the Board of Deans of the Faculty of Electrical Engineering. 